\title{
Combined RF-based drone detection and classification
}

\author{
Sanjoy Basak*, Sreeraj Rajendran ${ }^{\dagger}$, Sofie Pollin ${ }^{\dagger}$, and Bart Scheers* \\ Email: \{sanjoy.basak, bart.scheers\}@rma.ac.be, \\ \{sreeraj.rajendran, sofie.pollin\}@esat.kuleuven.be \\ ${ }^{*}$ Department CISS, Royal Military Academy, Belgium, ${ }^{\dagger}$ Department ESAT, KU Leuven, Belgium
}

\begin{abstract}
Despite several beneficial applications, unfortunately, drones are also being used for illicit activities such as drug trafficking, firearm smuggling or to impose threats to security-sensitive places like airports and nuclear power plants. The existing drone localization and neutralization technologies work on the assumption that the drone has already been detected and classified. Although we have observed a tremendous advancement in the sensor industry in this decade, there is no robust drone detection and classification method proposed in the literature yet. This paper focuses on radio frequency (RF) based drone detection and classification using the frequency signature of the transmitted signal. We have created a novel drone RF dataset using commercial drones and presented a detailed comparison between a two-stage and combined detection and classification framework. The detection and classification performance of both frameworks are presented for a single-signal and simultaneous multi-signal scenario. With detailed analysis, we show that You Only Look Once (YOLO) framework provides better detection performance compared to the Goodness-of-Fit (GoF) spectrum sensing for a simultaneous multi-signal scenario and good classification performance comparable to Deep Residual Neural Network (DRNN) framework.
\end{abstract}

Index Terms-signal detection and classification, sensor systems and applications, UAV.

\section{INTRODUCTION}

$\mathbf{T}$ HERE has been a tremendous technological improvement in the drone industry. Drones are now being equipped with state-of-the-art (SoA) technologies and sensors such as GPS, LIDAR, radar and visual sensors. These technologies facilitate drones to support numerous applications like cinematography, farming, surveillance and recreational activities. Drones equipped with advanced technologies have great potential for damaged infrastructure inspections, urgent aid supply, search and rescue operations to remote and unreachable places. Apart from these beneficial applications, drones are also being used for illegal activities which impose risks to public safety. The illegal activities include but are not limited to violation of public privacy, drug trafficking, firearm smuggling, bombing, and invading security-sensitive places like airports and nuclear power plants.

Several Counter Unmanned Aircraft Systems (C-UAS) have been proposed to disable the attack from a drone, which are mainly divided into two categories: hard and soft interception (kinetic or non-kinetic solution). The kinetic solutions include intercepting a drone using (i) a trained bird of prey (ii) a net gun [1] (iii) a laser beam, and (iv) a firearm. The non-kinetic solutions include: (i) GPS spoofing [1] to deceive a drone's localization system and (ii) RF jamming. Irrespective of the chosen solution for any environment, the presence of a drone should be detected and classified beforehand.

Detecting and classifying a drone automatically is a challenging task. Some popular technological approaches to detect and classify a drone include: (i) Radar detection, (ii) Video detection, (iii) Acoustic detection, and (iv) RF-based detection. A comprehensive literature review on the current SoA Machine Learning-based drone detection and classification using these technologies is presented in [2]. Researchers also proposed to integrate multiple technologies [3] for the detection and classification of UAVs.

The radar detection exploits the back-scattered RF signal to detect and classify a drone. The conventional radar systems will fail to detect a mini-drone due to its small radar cross section (RCS). To overcome this problem, researchers utilized the micro-Doppler signature of a Quadcopter or a Multi-rotor UAV to detect and classify it using a multi-static radar [4] or a Frequency Modulated Continuous Wave (FMCW) radar [5,6]. A complete review of the detection and classification strengths of the current SoA FMCW radars is presented in [6].

The video/image detection includes both visual and thermal detection, and in [7]-[10] researchers proposed several drone detection methods using this technology. With this technique, drone detection is performed by analyzing its color, shape and edge information [7]. The detection method is reliable, however, it requires a line of sight (LOS) between the drone and camera and the performance is highly dependent on daylight conditions and weather conditions like dust, rain, fog and cloud. Furthermore, the resemblance of a bird to a drone makes it more challenging for a video detector. In [10], the authors utilized the motion and trajectory information of a drone to differentiate it from a bird. A brief overview of the frameworks capable of differentiating a drone from a bird is presented in [11].

The acoustic detection system utilizes the sound generated by flying drones to detect its presence using microphones. In [12], the authors proposed a framework using Hidden Markov Models (HMM) to perform phoneme analysis and identify a flying drone from its emitted sounds. Furthermore, the detection and tracking of a drone using an array of antennas are also proposed in the literature. A small tetrahedral array [13] or a microphone array consisting of 120 elements [14] are used for drone detection and tracking. The acoustic detection generally works well in a quiet or less noisy environment, 
however, the performance deteriorates if the environment is noisy such as urban or industrial areas or near seashores.

One of the most promising approaches to detect the presence of a drone is through RF sensing. The commercial drones perform RF communication with their ground control station (GCS) for flight control and navigation, live video transmission and transfer telemetry information. The autonomous drones also perform active RF communication to transfer live videos and telemetry messages. An RF drone detection system can detect a drone by monitoring the communication frequency spectrum. There are a few RF-based drone detection techniques proposed in the literature [15]-[20]. In [15], the presence of a drone is detected by monitoring how frequently the data packet is being transmitted at $2.4 \mathrm{GHz}$. Since most of the drones use different non-standardized protocols for their communication with their controller, the data packet transmission rate varies from $\mathrm{WiFi}$ and other Access Points (AP) [15]. In [16], the detection is performed by measuring the data packet length of a drone's communication link. These detection methods are inefficient since the detector can be easily spoofed by an application communicating with an AP with the same packet transfer rate or having the same packet length as a drone. In [17], a WiFi based drone surveillance method is proposed, where the identification is performed by a $\mathrm{WiFi}$ statistical fingerprinting technique. In [21], we observed that several commercial drone's GCS uses Frequency Hopping Spread Spectrum (FHSS) transmission as the radio control (RC) signal, which should also be accounted for in the identification method.

The detection and identification of a drone using the frequency signatures is presented in $[18,19]$, using Deep Neural Network (DNN) based classifiers. In [18], the author developed a dataset using three commercial drones and used a simple feedforward DNN to detect and identify them. In [19], the author presented the detection, identification and classification on the same dataset using a Convolutional Neural Network $(\mathrm{CNN})$. These studies were performed on a limited dataset and the impact of noise on the detection performance was not studied. Moreover, the detection performance in presence of multiple signals or interference was not investigated.

We presented an RF-based drone detection using GoF spectrum sensing and DoA estimation using MUSIC algorithm in [22]. Drone signal detection using wideband CFARbased energy detection and the feature extraction performance is presented in [21]. In [23], we presented drone signal classification using a DRNN framework. The classification was performed assuming a signal is already detected by a spectrum sensing algorithm. A complete solution for drone detection and classification based on RF fingerprints was not presented in our previous works, which we address in this paper. We propose two complete solutions for drone signal detection and classification, and provide an in-depth performance comparison. The detection and classification is performed in two different ways: (i) Two-stage detection and classification process: where the signal detection is initially performed using an efficient spectrum sensing method. This will detect all of the signals present in the spectrum. The detected signals are passed to a SoA classifier to provide a robust classification. (ii) Combined detection and classification: where the signals are detected and classified simultaneously. For both proposed methods, we perform detection and classification with the received signal from a single receiver and by using frequency domain fingerprints. The advantages are (i) The possibility of using the received signal from a single receiver eliminates the requirement of calibration of multiple receivers. This makes both methods easily deployable with a low cost SDR and a computational unit. (ii) The frequency domain detection and classification provide the necessary information for a possible RF jammer. Both methods can perform fast detection and classification, even in the presence of overlapping signals both in the time and frequency domain. They are more generalized and robust as they understand the position and type of signal.

The main contributions of this paper are the following:

1) A novel and realistic multi-signal dataset is created using nine commercial drones and non-drone signals (i.e. WiFi communication signals). The dataset will be made public for future research.

2) The YOLO-lite architecture is recreated from scratch and modified to perform the combined drone signal detection and classification. The two-stage detection and classification is performed using GoF spectrum sensing and DRNN classifier.

3) The simultaneous multi-signal detection, spectrum localization and classification in the ISM band is presented in this paper. We are the first to propose a framework for simultaneous multi-signal drone detection and classification.

4) The detection and classification performance of both frameworks are evaluated on our dataset. Through detailed comparisons, we show that the YOLO-lite framework provides better detection performance compared to the GoF sensing and a good classification performance comparable to the DRNN classifier.

The rest of the paper is organized as follows: A mathematical model of the received signal in an ISM band is presented in section II. Section III provides an overview of the SoA techniques for two-stage and combined signals detection and classification. The technical details to perform two stage, and combined detection and classification is presented in section IV. The dataset development and the experiment strategies are explained in section $\mathrm{V}$. The performance analysis is presented in section VI and the concluding remarks are provided in section VII.

\section{Problem STATEMENT}

The ISM bands are generally populated by several homogeneous and heterogeneous RF transmissions. The transmitters generally use spread spectrum technology to perform the communications. The FHSS transmissions are generally blind whereas the Direct Sequence Spread Spectrum (DSSS) transmissions are often cognitive in nature. Most commercial drones use DSSS signal for video signal transmission. Unlike FHSS transmissions, they perform sensing to find a free (or relatively free) channel before starting to transfer video signal. One example of such heterogeneous transmission at $2.4 \mathrm{GHz}$ 
is shown in Fig 1. As it can be seen from the figure, four transmissions are occurring at the same time where three transmitters use the DSSS technology and one transmitter uses the FHSS technology.

The received signal from a drone can be expressed as:

$$
r(t)=\sum_{k=1}^{k=K} y_{k}(t) * h_{k}(t)+n(t),
$$

where, $y_{k}(t)$ is complex baseband transmitted signal, $h_{k}(t)$ is the time varying impulse response, $k$ denotes the index of transmitted signal, $\mathrm{K}$ is the total number of available transmitters in the ISM band, $n(t)$ is the Additive White Gaussian Noise (AWGN). With the help of discrete Fourier transform (DFT), the complex time domain receive signal can be converted into $M_{t}$ consecutive segments, each with length $N_{f}$ (= FFT size). The magnitude of the DFT matrix gives us the spectrogram matrix. This study aims to detect and classify drone and WiFi communication signals from the spectrogram matrix. The spectrogram representation provides more information compared to a Power Spectral Density (PSD) or IQ representation of the signal. It enables the classifier to determine useful RF signal features like frequency, bandwidth, dwell time and hop rate. Since commercial drones use pseudorandom number generators to generate the communication signals, the hopping pattern or the signal position will vary. The objective of our work is not to learn the hopping pattern, but rather to learn how the data/signal is distributed in the spectrum to detect and classify them. Deep Learning (DL) algorithms learn the signal distribution which is dependent upon factors like: (i) frequency, (ii) bandwidth, (iii) modulation, (iv) filtering parameters (v) device nonlinearities etc. We aim to utilize the DL algorithm to learn these factors from the spectrogram matrix.

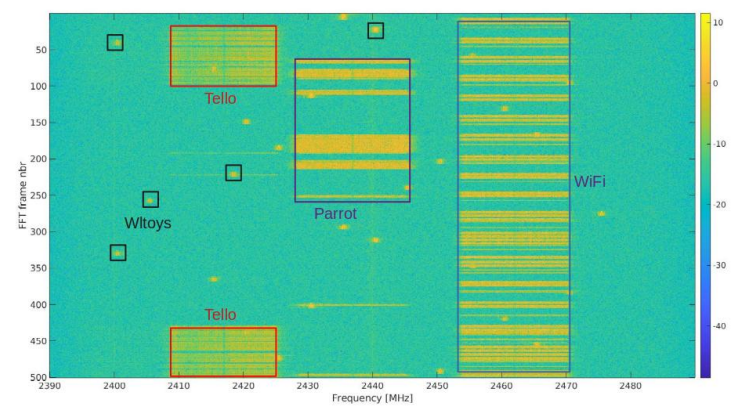

Fig. 1: Presence of multiple signals at $2.4 \mathrm{GHz}$ ISM band

\section{BACKGROUND}

\section{A. Signal Detection}

The conventional spectrum sensing methods can be classified into parametric and non-parametric methods. The parametric methods require prior knowledge of the transmitted signal for the detection, whereas the non-parametric methods also known as blind sensing do not require any knowledge of the signal. Most popular spectrum sensing methods are energy detection, eigenvalue detection, matched filter and cyclostationary feature detection [24]. The energy detection and eigenvalue detection are non-parametric methods. The energybased detection have been widely used for decades mainly due to its simplicity. The signal is detected if the measured energy is greater than the threshold corresponding to the specified false alarm rate. The eigenvalue detection method estimates the ratio of maximum and minimum eigenvalue and compares it with the threshold to determine if any signal is present. The cyclostationary and matched filtering are parametric methods, they require perfect knowledge of the transmitted signal and can work better at lower signal-to-noise ratio (SNR) compared to the energy-based detection [25]. The cyclostationary spectrum sensing method exploits the periodicity introduced in the transmitted signal. The matched filtering method detects a signal by correlating a known template (extracted from the transmit signal) with the received signal. Both methods are targeted towards specific (or known) signals and require a high computational cost.

For the drone signal detection within two-stage detection and classification, we choose wideband GoF based blind spectrum sensing algorithm [26]. The GoF sensing can provide better performance compared to the conventional energy detection using fewer samples of the received signal, at low SNR conditions and in presence of non-Gaussian noise [27]. The wideband GoF sensing uses DFT to divide the frequency band into small frequency bins and perform narrowband GoF sensing on each bin. In this paper, we have used Andersondarling test statistic for the GoF sensing [26].

\section{B. Signal classification}

DL methods have shown SoA performances in the classification of wireless signals and outperformed the conventional classification methods. Some remarkable works have been published $[28,29]$ in the past few years regarding the classification of modulated signals and device fingerprinting using merely the raw received signals. In the recent years, CNN frameworks have been widely investigated for wireless signal recognition and classification problems [28,30]. Among different variants of CNNs, the residual network-based CNN [31] have shown great performance and outperformed other classifiers with equivalent network depth. In this paper, we adapt the DRNN proposed in [28] for the drone and $\mathrm{WiFi}$ signal classification.

\section{Combined Detection and Classification}

The DNN based visual object detection and classification techniques provide great tools such as YOLO[32] for the combined RF signal detection, frequency localization and classification. Signal detection/classification from a spectrogram image is analogous to the visual object detection and classification. A spectrogram image provides the time and frequency information of a spectrum instance, which can be utilized to perform the detection and classification. Since we are interested in wideband signals, the localization and bounding box will also enable to determine important features from the detected signal, such as center frequency, bandwidth, dwell 
time and hop interval. Such information can be used within a cognitive radio to perform dynamic spectrum access functionality and avoid collisions in a spectrum sharing environment. YOLO was first used in [33] to perform signal detection and frequency localization. In [34], WiFi and LTE signal detection, feature extraction and classification was performed using a pretrained YOLO framework. In this paper, we develop a YOLO framework from scratch to perform the combined drone RF signal detection and classification on the spectrogram image.

\section{TEChNiCAL ApProach}

\section{A. Two stage detection and classification}

1) Signal detection: The signal detection is performed using the Anderson-Darling (AD) GoF test [26]. The complex time-domain receive signal is converted into the frequency domain using an N-point DFT operation on $\mathrm{K}$ consecutive segments. This results in a sequence of $\mathrm{X}_{k}$ of length $\mathrm{K}$ for every frequency bin. We perform a hypothesis test using an $\mathrm{AD}$ tester for each frequency bin to decide whether only noise or a signal is present in the bin. We assume, there is only noise present in the frequency bin if the normalized power spectral coefficient $\left(\frac{2\left|X_{k}\right|^{2}}{N \sigma^{2}}\right)$ follows a $\chi^{2}$ distribution. The length of DFT is $\mathrm{N}$ and $\sigma^{2}$ is the noise power. We estimated the noise power by exploiting the histogram of the power spectrum [35].

The $\mathrm{AD}$ test statistic $\left(A_{n}^{2}\right)$ is calculated for each frequency bin as:

$$
A_{n}^{2}=-n-\frac{\sum_{i=1}^{n}(2 i-1)\left(\ln z_{i}+\ln \left(1-z_{(n+1-i)}\right)\right)}{n}
$$

with $z_{i}=F_{o}\left(x_{i}\right)$

Here, $F_{o}$ represents the Cumulative Distribution Function (CDF) of a chi-square distribution with 2 degrees of freedom, $x_{1} \leq x_{2} \leq \ldots \leq x_{n}$ are the samples under test and $\mathrm{n}$ represents the total number of samples.

If $A_{n}^{2}>\lambda$, we assume the signal is present in the frequency bin, otherwise, we assume that the frequency bin only contains noise. Here, $\lambda$ corresponds to the detection threshold. A detailed explanation of the AD GoF sensing, the derivation of $A_{n}^{2}$ and the procedure to perform the hypothesis test on complex received signals from a Software Defined Radio (SDR) are provided in [26,27]. The value of $\lambda$ is determined considering 5\% False Alarm Rate (FAR). The suitable value for $\lambda$ was calculated through the AD GoF tests on the drone dataset. The value of $\lambda=3.89$ provided us an approximate $5 \%$ FAR.

2) Signal classification: The classification is performed using an adaptation of the DRNN framework proposed in [28]. The architecture is depicted in Table I and the building blocks are shown in Fig 2. The DRNN framework consists of $\mathrm{N}$ residual stack units, two fully connected (FC) layers and a softmax layer. For all convolution operations, we have used 32 filters with a kernel size of $3 \times 3$, apart from the first layer of the residual stack where the kernel size is $1 \times 1$. For the max pooling, a kernel size of $2 \times 2$ is used with a stride factor of 1. For each FC layer, we have employed a scaled exponential linear unit (SeLU) activation and mean response scaled initialization [36]. To prevent overfitting, we have performed $50 \%$ dropout after each FC layer. A softmax activation is used at the final layer to give the prediction probability. We haven't performed any batch normalization operation, since, we did not observe any additional improvement with it in the classification performance.

If any signal is detected from the spectrum sensing algorithm, the complete spectrum (i.e. the time domain RX signal) is passed to the classification stage. The time-domain signal is converted to a spectrogram signal of size $256 \times 256$ and the classification is performed on the signal. Since we are interested in comparing the classification performance with the YOLO framework, we kept the input size the same for both algorithms.

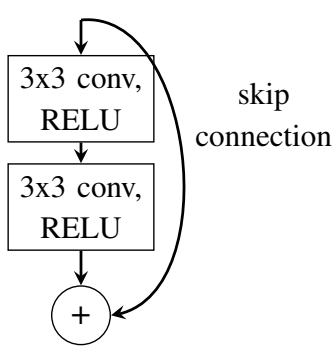

(a) ResUnit

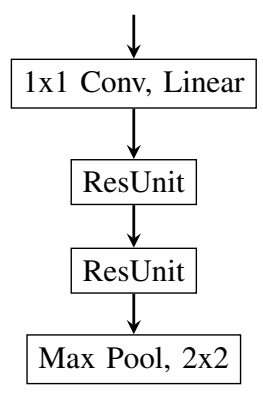

(b) Residual stack
Fig. 2: Building blocks of the deep residual network architecture

TABLE I: DRNN architecture

\begin{tabular}{|c|c|c|}
\hline Layer no. & Layer name & Output dimensions \\
\hline 1. & Input & $256 \times 256$ \\
\hline 2. & Residual stack & $128 \times 128 \times 32$ \\
\hline 3. & Residual stack & $64 \times 64 \times 32$ \\
\hline 4. & Residual stack & $32 \times 32 \times 32$ \\
\hline 5. & Residual stack & $16 \times 16 \times 32$ \\
\hline 6. & Residual stack & $8 \times 8 \times 32$ \\
\hline 7. & FCL/SELU & 128 \\
\hline 8. & FCL/SELU & 128 \\
\hline 9. & FCL/Softmax & 10 \\
\hline
\end{tabular}

\section{B. Combined detection and classification with YOLO}

We implement one of the variants of the YOLO architecture to perform the simultaneous detection and classification of drone and $\mathrm{WiFi}$ communication signals. One of the biggest strengths of the YOLO framework is that it can detect the signal, determine spectral features like frequency, bandwidth, dwell time and predict the class of the detected signal simultaneously. The raw spectral power values of the RF signal in time and frequency domain are used as the input. Since recognizing such time-frequency domain spectral events are relatively simpler than the visual object recognition [33], a smaller network may be sufficient for the YOLO detection and classification task. In our experiments, we have adapted the YOLO-lite [37] architecture, which is a smaller and faster network and can be deployed on a non-GPU computer. Our adaptation of the YOLO-Lite architecture is shown in Table II. We have used leaky-relu activation after each convolution operation $(\mathrm{C} 1-\mathrm{C} 6)$ and linear activation on the $\mathrm{C} 7$ layer. 
The max-pooling operation is performed after the convolutions (C1 - C5). Finally, a fully connected layer is employed, and sigmoid activation is performed.

TABLE II: YOLO Network architecture

\begin{tabular}{|c|c|c|}
\hline Layer & Filters & Size/Stride \\
\hline Conv2D (C1) + Max Pool (MP) & 16 & $3 \times 3 / 1+2 \times 2 / 2$ \\
\hline C2 + MP & 32 & $3 \times 3 / 1+2 \times 2 / 2$ \\
\hline C3 + MP & 64 & $3 \times 3 / 1+2 \times 2 / 2$ \\
\hline C $4+$ MP & 128 & $3 \times 3 / 1+2 \times 2 / 2$ \\
\hline C5 + MP & 128 & $3 \times 3 / 1+2 \times 2 / 2$ \\
\hline C6 & 256 & $3 \times 3 / 1$ \\
\hline C7 & 125 & $1 \times 1 / 1$ \\
\hline FCL/Sigmoid activation & & \\
\hline
\end{tabular}

A spectrogram dataset with a dimension of $256 \times 256$ is used as the input. The network produces an output grid containing the detection probability, bounding box coordinates and class probabilities as shown following:

$$
\mathrm{O} / \mathrm{P} \text { shape }=\mathrm{S} \times \mathrm{S} \times(\mathrm{B} \times[\mathrm{C}, \mathrm{x}, \mathrm{y}, \mathrm{w}, \mathrm{h}]+\mathrm{P})
$$

Here, $\mathrm{S}$ denotes the size of the grid. Each grid cell contains B bounding boxes, the confidence score of the detection: c, 2D coordinates: $\mathrm{x}, \mathrm{y}$, width: $\mathrm{w}$, height: $\mathrm{h}$ of the object and the class probability $\mathrm{P}$. We have used grid size 16, 2 bounding boxes and 10 different classes (Table III) for our tests. In the original YOLO-lite architecture output grid size of 8 was used, however, we found during our tests that in order to annotate the DSSS spectrograms (e.g. Tello, Parrot), a higher number of grid size is required. With the above specified parameters, the output dimension becomes $16 \times 16 \times 20$. We have used adam optimizer [38] to optimize the training loss. The training loss involves the minimization of the sum of mean squared error loss between the ground truth and network prediction. The complete training loss function provided in [32] is used for the training optimization.

\section{EXPERIMENTS}
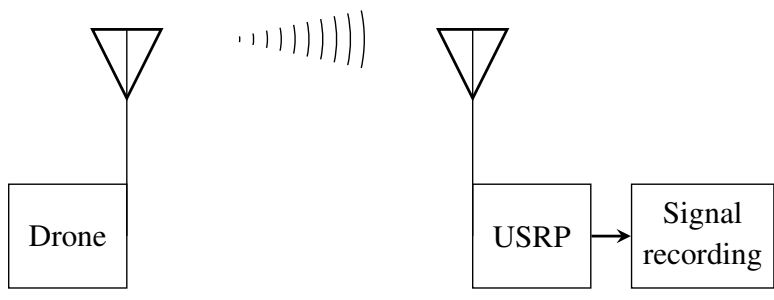

Fig. 3: Signal recording schematic

1) Experimental setup: The drone and WiFi signals were recorded in an anechoic chamber. For this experiment, we only considered the transmitters operating at $2.4 \mathrm{GHz}$. A universal software radio peripheral (USRP) X310 was used with an omnidirectional antenna. The receive sampling rate of 100 MSps was used to receive instantaneously from the complete $2.4 \mathrm{GHz}$ ISM band. Nine commercial radio controllers with drones and two WiFi routers (Table III) were used to develop

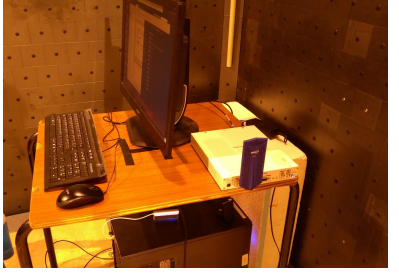

(a) Signal recording wih USRP

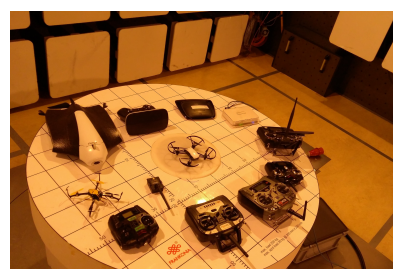

(b) Drones, GCS and WiFi routers
Fig. 4: Test setup in an anechoic chamber

TABLE III: Drones, Radio Controllers and WiFi sources used for the dataset development

\begin{tabular}{|c|c|c|c|}
\hline Nr. & Name & Signal Type & Freq(GHz) \\
\hline 1 & Parrot Disco & RC+Video & 2.4 \\
\hline 2 & Q205 & Video & 2.4 \\
\hline 3 & Tello & RC+Video & 2.4 \\
\hline 4 & MultiTx & RC & 2.4 \\
\hline 5 & Nineeagles & RC & 2.4 \\
\hline 6 & Spektrum DX4e & RC & 2.4 \\
\hline 7 & Spektrum DX6i & RC & 2.4 \\
\hline 8 & Wltoys & RC & 2.4 \\
\hline 9 & Taranis Q X7 (S500) & RC & 2.4 \\
\hline 10 (i) & Linksys router & IEEE802.11b/g & 2.4 \\
\hline 10 (ii) & Netgear router & IEEE802.11n & 2.4 \\
\hline
\end{tabular}

the dataset. The devices were placed seven meters apart from the receiver. Since a UAV controller generally uses a pseudorandom generator to generate the FHSS sequences as the RC signal, we included all possible hop sequences from each controller in our database. The controllers were turned off and on several times during data collection to inspect if the hop position changes and include that as well in our database.

2) Dataset development: To test the classification performance at lower SNRs, we introduced AWGN to the signal in the simulation environment. Generally, the SNR is calculated in the time domain by measuring the transmission power of the signal. Since the signal bandwidth of different drones is different, it becomes difficult to calculate the SNR in the time domain. Therefore, we calculated the signal SNR in the frequency domain as shown in Fig. 5a. Since the bandwidth and transmission power are different for different drones, the calculated SNR was also slightly different for different noise values as presented in Fig. 5b. To keep the performance analysis of different frameworks on the dataset simple, we considered the average SNR for the introduced AWGN values as shown in Fig. 5c.

In [23], we evaluated the classification performance in Rician and Rayleigh fading simulation environment where the classifier was trained with AWGN faded dataset. We did not observe any significant deviation in the classification performance due to the channel variation. Therefore, in this paper, we only evaluated the classification performance under AWGN conditions.

3) Implementation details: The GoF sensing was implemented in Matlab. The DRNN framework was implemented using Tensorflow-Keras and the YOLO-lite framework was implemented using TFlearn in Python, both running on top of Tensorflow [39]. The simulations and the neural network 


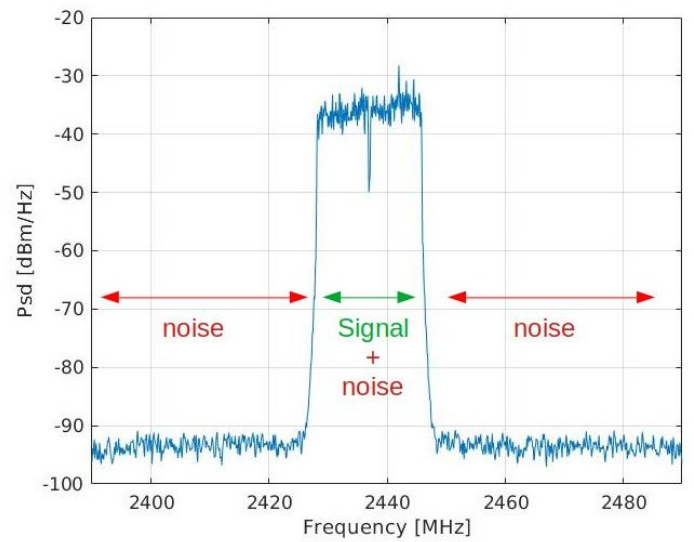

(a) SNR calculation in frequency domain

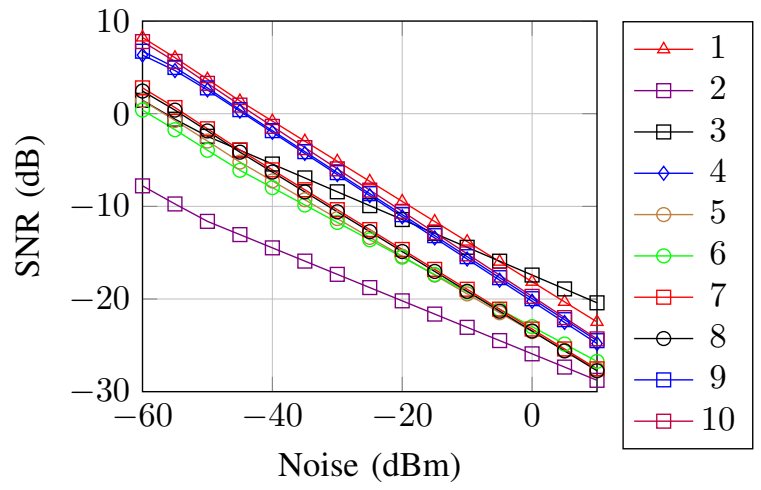

(b) Relation between noise vs SNR. The legends correspond to the device no. given in Table III

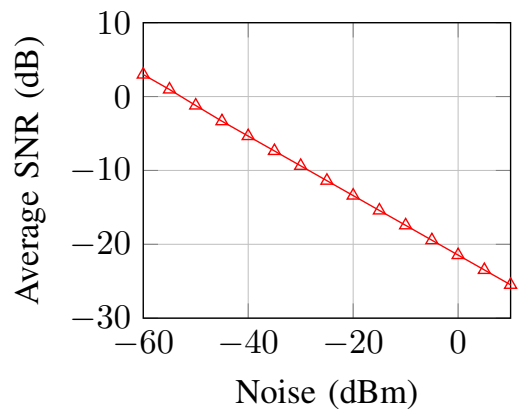

(c) Noise vs average SNR

Fig. 5: SNR variation using AWGN in the simulation environment

training and testing were performed on an Intel Core i7 computer equipped with Nvidia RTX 2080 GPU. The adam optimizer with a learning rate of 0.001 was used for the training optimization for both networks. The training was performed with a batch size of 32 .

\section{Performance analysis}

The performance analysis was performed for two scenarios: (i) detection and classification in presence of one signal at a time under AWGN conditions, (ii) detection and classification in presence of multiple signals simultaneously with frequency overlapped and non-overlapped cases.

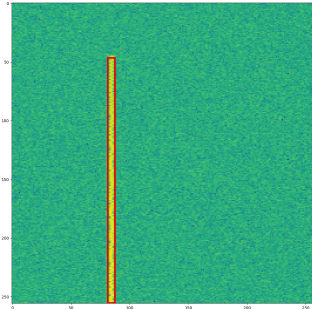

(a) DX4E

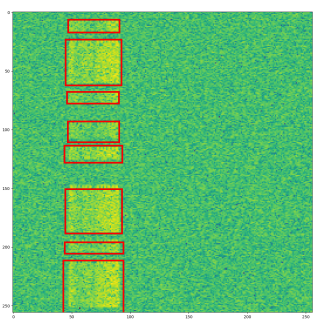

(c) Tello

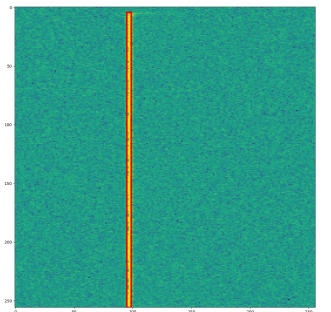

(b) DX6i

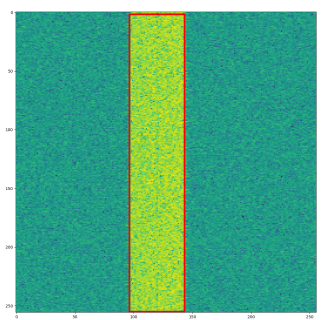

(d) Parrot
Fig. 6: Signal detection with YOLO-Lite

\section{A. Single signal detection and classification}

Signal detection and spectrum localization with the YOLOlite framework are presented in Fig. 6. As Fig. 6 shows, the signals were detected, localized in the spectral domain and classified by the framework accurately. The average probability of detection (PD) of YOLO under different SNR conditions is presented in Fig. 7a. A detection from the YOLOLite prediction is considered to be true if it satisfies the following two conditions: (i) the confidence score of any bounding box is greater than the specified threshold (i.e. C $>0.4$ ) and (ii) the Intersection over Union (IoU) is greater or equal to 0.50 (i.e. IoU $\geq 0.50$ ). The PD for the GoF test is calculated by comparing the true frequency bins with the predicted frequency bins from the AD test. As Fig. 7a displays, the PD from YOLO is comparable with the GoF test. The detection probability increases for both frameworks as the SNR increases. The YOLO PD saturates around 96\%, where it reaches at around $-3 \mathrm{~dB}$ SNR. This saturation happens because YOLO often could not detect all closely spaced hops of signals from Tello, Parrot and WiFi. One example of such a spectrum is shown in Fig. 6c. The GoF test also provides around $96 \%$ PD around SNR $-3 \mathrm{~dB}$, however, it increases further and reaches $99.9 \%$ at SNR $3 \mathrm{~dB}$.

To evaluate the classification performances, we used the F1-score parameter, which is a harmonic mean of precision and recall. Since the F1-score takes both precision and recall into account, it allows us to compare the performance of different classifiers using just one metric. The performance of classification with the YOLO and DRNN framework are plotted in Fig. 7b. In order to compare the classification performance of YOLO with the DRNN, we performed the 


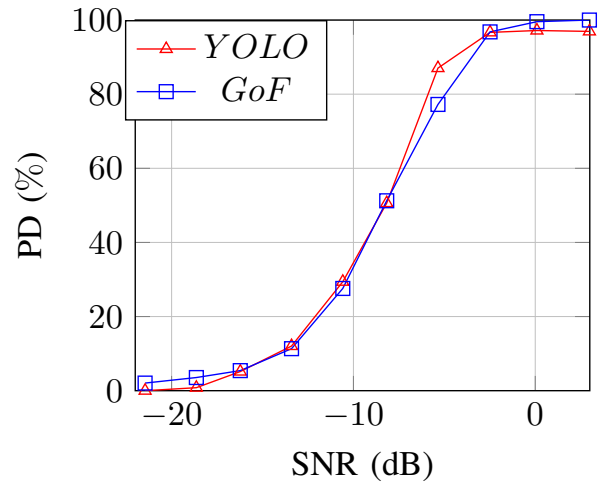

(a) Detection performance

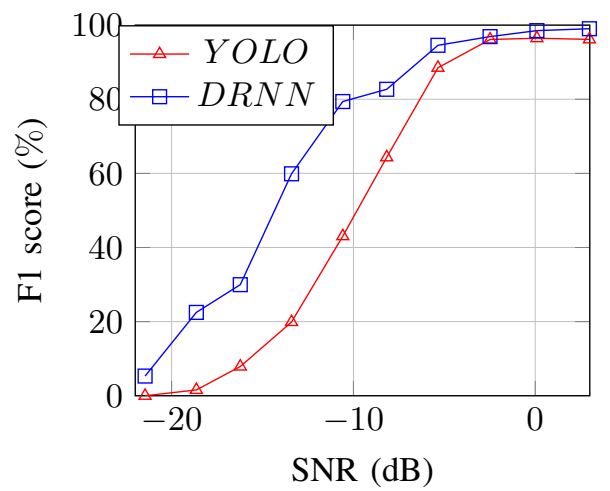

(b) Classification performance

Fig. 7: Detection probability and classification performance under AWGN conditions with YOLO and DRNN framework. Train samples: $4.9 \mathrm{k}$, Test samples: $2.1 \mathrm{k}$

classification with the DRNN framework independent of the GoF detection. At lower SNR, DRNN provides a better F1score compared to YOLO. This is expected since YOLOlite is much shallower framework compared to the DRNN framework. The F1-scores increases with the increase in SNR for both frameworks. The F1-score reaches approx. 97\% at SNR $-3 \mathrm{~dB}$ for both frameworks. The F1-score from the YOLO-lite saturates around 97\% at higher SNRs. The F1score from the DRNN framework increases to $99 \%$ at SNR 3 dB.

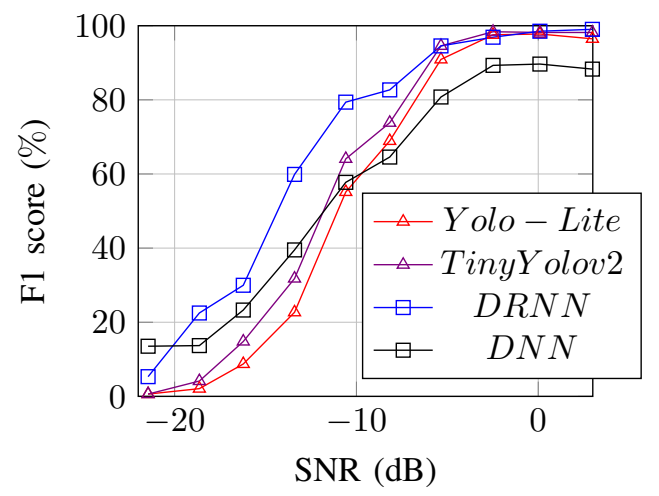

Fig. 8: Classification comparison with other frameworks under AWGN conditions. Train samples: $4.9 \mathrm{k}$, Test samples: $2.1 \mathrm{k}$
The classification performance of our YOLO-Lite and DRNN framework is compared with other existing frameworks namely the DNN framework proposed in [18] and the TinyYOLOv2-VOC framework [37]. The F1-scores of the classification performance under AWGN conditions are plotted in Fig. 8. The DRNN framework provided the best classification performance compared to the other frameworks. The TinyYOLOv2 provided slightly better classification performance compared to the YOLO-Lite framework. This is expected since it is a slightly deeper framework compared to the YOLO-lite framework. We also recreated Tiny-YOLOv2 from scratch for this classification test. The DNN model provided lower F1score compared to other frameworks from SNR $-10 \mathrm{~dB}$ and onwards. The F1-score of this framework was saturated at around $89 \%$, whereas YOLO-lite, Tiny-YOLOv2 and DRNN provided around $97 \%, 98 \%$ and $99 \%$ F1-score respectively at higher SNRs.

\section{B. Simultaneous multi-signal detection and classification}

In order to test the detection and classification performance in a simultaneous multi-signal scenario, the signals were added in the simulation environment as shown in Fig 9. To ensure the number of specified signals present in the spectrogram, we performed spectrum sensing before adding the RX signals. Each signal burst was a vector of $256 * 256=65536$ complex samples in the time domain. Later AWGN was introduced after adding the signals and it was converted to the frequency domain. The detection and classification from YOLO-lite is shown in Fig 10. In order to observe the prediction accuracy, the ground truth and prediction are plotted side by side and the true and predicted classes are also annotated in white color. As it can be seen from Fig 10, the signals were detected, localized in spectral domain and classified accurately. YOLOlite could also detect and classify signals which are overlapped in the frequency domain. It can be observed from Fig 10b, that WiFi and tello signals are overlapped in frequency domain. Similarly, DX6i and Parrot signals are also overlapped in the frequency domain. All of the signals are localized and classified accurately.

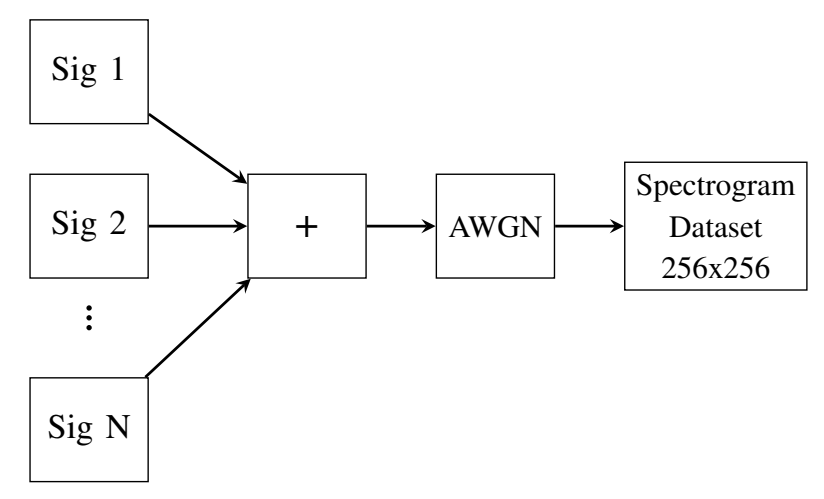

Fig. 9: Simulation schematic for multi-signal detection and classification

The multi-signal detection from the GoF sensing is shown in Fig 11. The spectrogram image is plotted in Fig. 11 (top) 

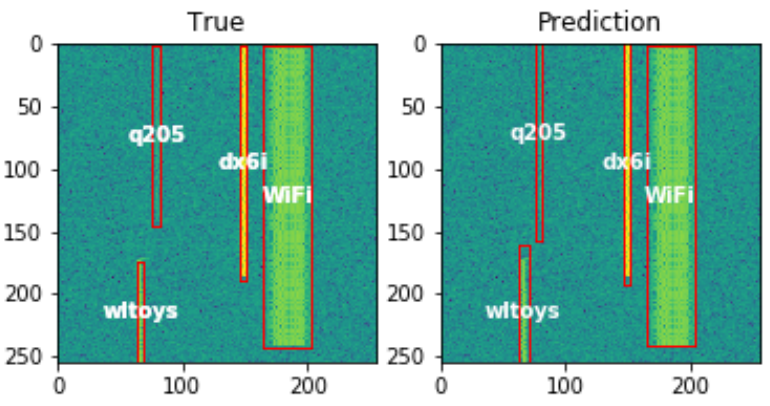

(a) Detection and classification in presence of 4 signals
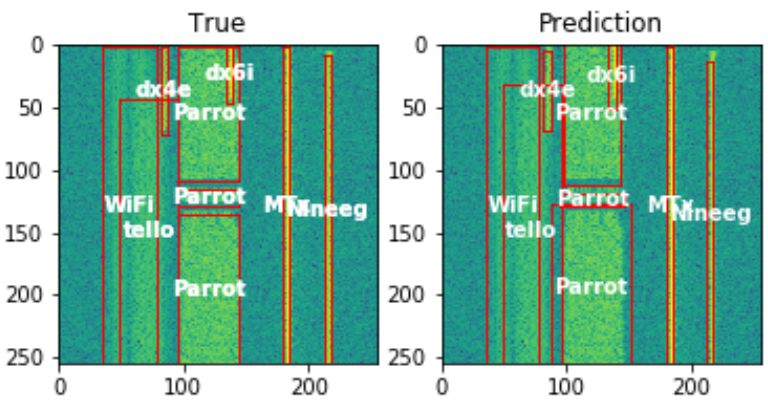

(b) Detection and classification in presence of 7 signals

Fig. 10: Detection and classification with YOLO in presence of multiple signals
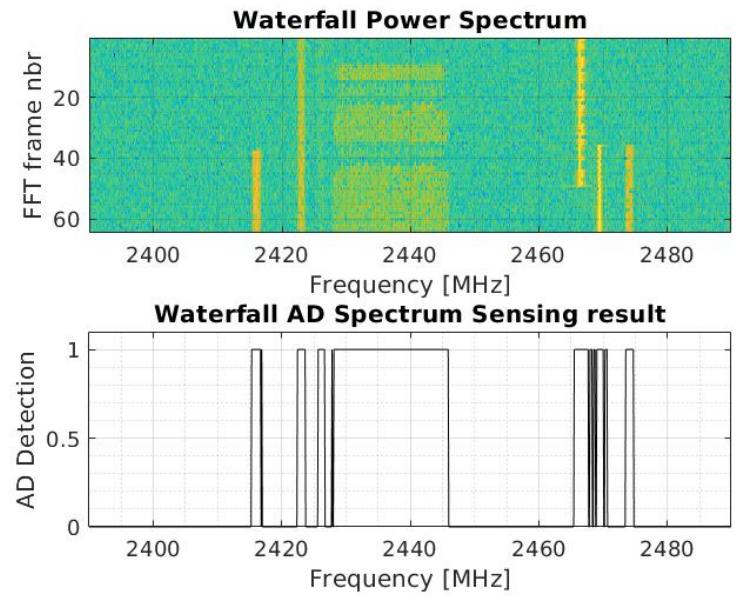

Fig. 11: Signal detection using GoF spectrum sensing in presence of multiple signals

and the AD GoF result is plotted in Fig. 11 (bottom). As it can be seen from the figure, that all seven signals are detected correctly.

The detection threshold for YOLO-lite was chosen to be 0.4. This threshold was chosen such that the maximum FA rate for the lowest SNR remains below 5\%. The threshold was kept the same for single and simultaneous multi-signal detection.

The PD of the GoF sensing and YOLO-lite over different SNRs are plotted in Fig. 12. As it can be seen from the figure, YOLO-lite showed better detection performance compared to the GoF sensing. For GoF sensing, the detection performance over different SNRs did not vary depending on the number of sources. On the contrary, an increase in the detection performance was observed with YOLO-lite. If we look at Fig. $5 \mathrm{~b}$, the SNRs for different signals are different. With wideband GoF sensing, if we have multiple signals with different SNR present in the spectrum, it impacts the noise floor estimation. This may result in an overestimation of the threshold. This issue was not observed with the YOLO-lite spectrum sensing.

The classification performance of DRNN and YOLO over different SNRs are plotted in Fig. 13. Similar to the PD, the F1-score of the YOLO classification increases with the increase in the number of sources. We can also observe the same phenomena with the F1-score of the DRNN classification. After detailed investigation, we found that the classification accuracy of any individual signal remains the same for single signal or simultaneous multi-signal scenario. At lower SNRs, the classification accuracy for different signals are generally different. There are two reasons behind this: (i) the actual SNR of the signals are not the same (Fig. 5b) (ii) classifiers can classify some signals better than other signals at lower SNRs. Fig. 5 b shows that the actual SNR of the signals is different for different classes. As it can be observed, one of the signals has a very low SNR compared to the other signals. This is because we added a constant AWGN noise and considered the average SNR for ease of analysis. When we calculate the average F1-score for the single-signal scenario, it averages equally having independent F1 scores for each class. However, for the multi-signal scenario, as the number of signals increases, the relatively lower F1 score of a particular class cannot make the average performance as worse as the single-signal scenario. Therefore, as the number of signals in the multi-signal scenario increases the average score also goes higher. Again, similar to the single drone scenario, DRNN showed better classification performance compared to YOLO.

$$
\triangle-N b=2-\nabla b=4-\nabla b=6 \multimap-N b=7
$$
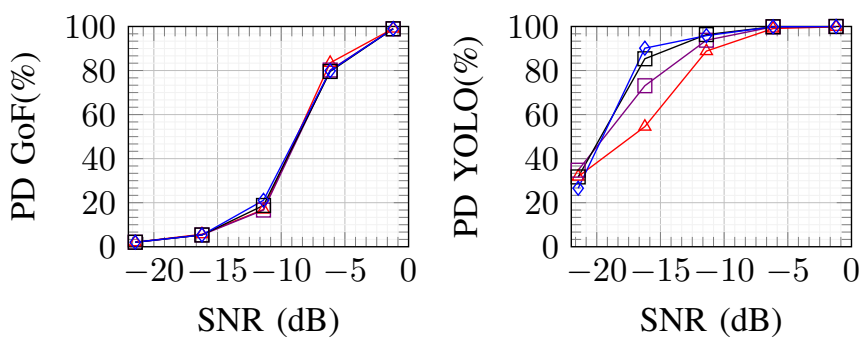

Fig. 12: Detection probability under AWGN conditions in presence of multiple-signals simultaneously. Train samples: $30.7 \mathrm{k}$, Test samples: $13.2 \mathrm{k}$

\section{Comparison summary}

The comparisons are summarized here:

- Signal detection: We have obtained better signal detection performance with YOLO compared to the GoF spectrum sensing on our dataset. At lower SNR regions, the GoF sensing showed high false alarm rate compared to the 


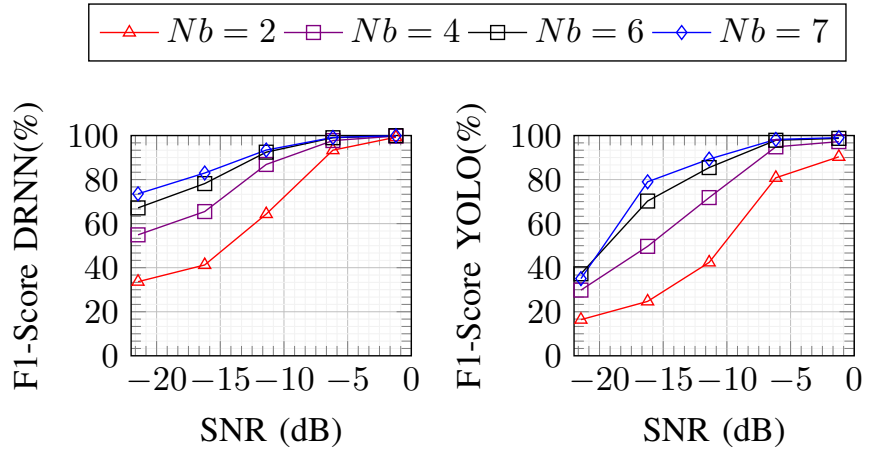

Fig. 13: Classification performance under AWGN conditions in presence of multiple-signals simultaneously. Train samples: $30.7 \mathrm{k}$, Test samples: $13.2 \mathrm{k}$

YOLO detection. Since YOLO is a supervised detection framework, the performance may deviate while detecting unknown signals. This issue can be resolved with transfer learning using a small labelled dataset of the new signal. On the contrary, the GoF sensing is a blind spectrum sensing method, it is able to detect any signal present in the spectrum.

- Signal classification: The DRNN framework provided better classification performance compared to the YOLO framework. It is expected from a deep residual network since it utilizes the skip connection feature in the architecture and the network is deeper than the YOLO framework.

- Signal localization and feature extraction: The signal localization and feature extraction is the best feature of the YOLO framework. The localization feature of YOLO enables us to detect multiple signals simultaneously and extract the useful features from the received signal. It can provide the center frequency, bandwidth, hop rate and dwell time of the detected signal. This information is required for an RF jammer to perform soft neutralization of a drone. With the two-stage detection and classification framework, it is not possible to extract all of these features. The DRNN framework does not provide the spectral position of the signal under classification. The GoF sensing provides the frequency and bandwidth of the signal, however, in presence of multiple signals, it is going to be difficult to associate the signal features with the classification label.

- Complexity analysis: A complexity analysis was performed to give an overview of the computational complexity and the inference time required for each framework. The total network parameter (that is, trainable + non trainable parameter), the mean inference time on total test samples and the mean prediction time per sample are presented in Table IV. The inference time calculation was performed with the dataset used in the single signal detection scenario, where the test sample size was $2.1 \mathrm{k}$ samples. We used a batch size of 100 and performed the test on Nvidia GeForce RTX 2080 Ti GPU. As it can be seen from Table IV, YOLO-lite is approximately 3.4 times faster than the DRNN framework.

The GoF spectrum sensing is a computationally simpler algorithm compared to the DRNN and YOLO-lite frameworks. It does not require any high-end computational unit. During the SafeShore [40] project, we implemented the GoF sensing using $\mathrm{C}++$ on an Odroid-XU4 platform and performed real-time tests.

TABLE IV: Complexity comparison

\begin{tabular}{|c|c|c|}
\hline Parameter & DRNN & YOLO Lite \\
\hline Total parameters & 469,322 & $41,537,149$ \\
\hline Mean inference time & $3.98 \mathrm{~s}$ & $1.16 \mathrm{~s}$ \\
\hline Mean prediction time per sample & $1.89 \mathrm{~ms}$ & $0.552 \mathrm{~ms}$ \\
\hline
\end{tabular}

- Limitation: One of the limitations of both proposed methods is the classification of completely unknown signals. Since the classification is performed in a supervised manner, the classifier may not be able to classify or provide a label to the transmitted signals from newer drones. There are two possible outcomes in such a case: (i) classifier will label it as an existing drone signal if the TX signal has a similar frequency fingerprint, (ii) classifier will be confused and provide a very low classification score for all classes. Similarly, some specific models of UAV controllers may use a completely different hopping sequence compared to another controller under the same model. The YOLO detection and classification performance for such cases are not yet tested. We are going to investigate and address these issues in our future work.

\section{CONCLUSION}

In this paper, we performed drone signal detection, spectrum localization and classification using two stages and combined detection and classification methods. Under the two-stage technique, we used the GoF sensing for the detection and the DRNN framework for the classification. The YOLOlite framework was recreated from scratch to perform the combined drone RF signal detection, spectrum localization and classification. A detailed performance comparison between both of the techniques is presented using a novel drone dataset that was prepared for this study. We obtained good detection and classification performances with both techniques. Since the classification is performed in a supervised manner, the performance may deviate in presence of unknown or newer drone signals which we mentioned in detail in the limitation discussion. In the future work, we are going to investigate the unsupervised scenarios, since we are interested in developing a robust framework that can detect and classify all drone signals irrespective of the dataset it is trained with.

\section{REFERENCES}

[1] D. Sathyamoorthy, "A review of security threats of unmanned aerial vehicles and mitigation steps," J. Def. Secur, vol. 6, no. 1, pp. 81-97, 2015.

[2] B. Taha and A. Shoufan, "Machine learning-based drone detection and classification: State-of-the-art in research," IEEE Access, vol. 7, pp. 138669-138682, 2019. 
[3] G. Ding, Q. Wu, L. Zhang, Y. Lin, T. A. Tsiftsis, and Y. Yao, "An amateur drone surveillance system based on the cognitive internet of things," IEEE Communications Magazine, vol. 56, no. 1, pp. 29-35, 2018.

[4] F. Fioranelli, M. Ritchie, H. Griffiths, and H. Borrion, "Classification of loaded/unloaded micro-drones using multistatic radar," Electronics Letters, vol. 51, no. 22, pp. 1813-1815, 2015. [Online]. Available: https://ietresearch.onlinelibrary.wiley.com/doi/abs/10.1049/el.2015.3038

[5] J. Drozdowicz, M. Wielgo, P. Samczynski, K. Kulpa, J. Krzonkalla, M. Mordzonek, M. Bryl, and Z. Jakielaszek, " $35 \mathrm{ghz} \mathrm{fmcw}$ drone detection system," in 2016 17th International Radar Symposium (IRS), 2016, pp. 1-4.

[6] A. Coluccia, G. Parisi, and A. Fascista, "Detection and classification of multirotor drones in radar sensor networks: A review," Sensors, vol. 20, no. 15, 2020. [Online]. Available: https://www.mdpi.com/1424$8220 / 20 / 15 / 4172$

[7] Z. Zhang, Y. Cao, M. Ding, L. Zhuang, and W. Yao, "An intruder detection algorithm for vision based sense and avoid system," in 2016 International Conference on Unmanned Aircraft Systems (ICUAS), 2016, pp. 550-556.

[8] S. R. Ganti and Y. Kim, "Implementation of detection and tracking mechanism for small uas," in 2016 International Conference on Unmanned Aircraft Systems (ICUAS), 2016, pp. 1254-1260.

[9] R. Stolkin, D. Rees, M. Talha, and I. Florescu, "Bayesian fusion of thermal and visible spectra camera data for mean shift tracking with rapid background adaptation," in SENSORS, 2012 IEEE, 2012, pp. 1-4.

[10] S. R. Ganti and Y. Kim, "Implementation of detection and tracking mechanism for small uas," in 2016 International Conference on Unmanned Aircraft Systems (ICUAS), 2016, pp. 1254-1260.

[11] A. Coluccia, A. Fascista, A. Schumann, L. Sommer, M. Ghenescu, T. Piatrik, G. De Cubber, M. Nalamati, A. Kapoor, M. Saqib, N. Sharma, M. Blumenstein, V. Magoulianitis, D. Ataloglou, A. Dimou, D. Zarpalas, P. Daras, C. Craye, S. Ardjoune, D. De la Iglesia, M. Mández, R. Dosil, and I. González, "Drone-vs-bird detection challenge at ieee avss2019," in 2019 16th IEEE International Conference on Advanced Video and Signal Based Surveillance (AVSS), 2019, pp. 1-7.

[12] M. Nijim and N. Mantrawadi, "Drone classification and identification system by phenome analysis using data mining techniques," in 2016 IEEE Symposium on Technologies for Homeland Security (HST), 2016, pp. 1-5.

[13] M. Benyamin and G. H. Goldman, "Acoustic Detection and Tracking of a Class I UAS with a Small Tetrahedral Microphone Array." Adelphi, MD, September 2014

[14] J. Busset, F. Perrodin, P. Wellig, B. Ott, K. Heutschi, T. Rühl, and T. Nussbaumer, "Detection and tracking of drones using advanced acoustic cameras," in Unmanned/Unattended Sensors and Sensor Networks XI; and Advanced Free-Space Optical Communication Techniques and Applications, ser. Society of Photo-Optical Instrumentation Engineers (SPIE) Conference Series, E. M. Carapezza, L. Laycock, H. J. White, P. G. Datskos, and C. Tsamis, Eds., vol. 9647, Oct. 2015, p. 96470F.

[15] P. Nguyen, M. Ravindranatha, A. Nguyen, R. Han, and T. Vu, "Investigating cost-effective rf-based detection of drones," in Proceedings of the 2nd Workshop on Micro Aerial Vehicle Networks, Systems, and Applications for Civilian Use, ser. DroNet '16. New York, NY, USA: Association for Computing Machinery, 2016, p. 17-22. [Online]. Available: https://doi.org/10.1145/2935620.2935632

[16] P. Kosolyudhthasarn, V. Visoottiviseth, D. Fall, and S. Kashihara, "Drone detection and identification by using packet length signature," in 2018 15th International Joint Conference on Computer Science and Software Engineering (JCSSE), 2018, pp. 1-6.

[17] I. Bisio, C. Garibotto, F. Lavagetto, A. Sciarrone, and S. Zappatore, "Blind detection: Advanced techniques for wifi-based drone surveillance," IEEE Transactions on Vehicular Technology, vol. 68, no. 1, pp. 938-946, 2019.

[18] M. Al-Sa'd, A. Al-Ali, T. Khattab, and A. Erbad, "Rf-based drone detection and identification using deep learning approaches: An initiative towards a large open source drone database," Future Generation Computer Systems, vol. 100, 052019.

[19] S. Al-Emadi and F. Al-Senaid, "Drone detection approach based on radio-frequency using convolutional neural network," in 2020 IEEE International Conference on Informatics, IoT, and Enabling Technologies (ICIoT), 2020, pp. 29-34.

[20] M. M. Azari, H. Sallouha, A. Chiumento, S. Rajendran, E. Vinogradov, and S. Pollin, "Key technologies and system trade-offs for detection and localization of amateur drones," IEEE Communications Magazine, vol. 56, no. 1, pp. 51-57, 2018.
[21] P. Stoica, S. Basak, C. Molder, and B. Scheers, "Review of counteruav solutions based on the detection of remote control communication," in 2020 13th International Conference on Communications (COMM), 2020, pp. 233-238.

[22] S. Basak and B. Scheers, "Passive radio system for real-time drone detection and doa estimation," in 2018 International Conference on Military Communications and Information Systems (ICMCIS), May 2018, pp. 1-6.

[23] S. Basak, S. Rajendran, S. Pollin, and B. Scheers, "Drone classification from rf fingerprints using deep residual nets," in 2021 International Conference on COMmunication Systems NETworkS (COMSNETS), 2021, pp. 548-555.

[24] H. Sun, A. Nallanathan, C.-X. Wang, and Y. Chen, "Wideband spectrum sensing for cognitive radio networks: a survey," IEEE Wireless Communications, vol. 20, no. 2, pp. 74-81, Apr. 2013, iNSPEC Accession Number: 13474041.

[25] I. Kakalou, D. Papadopoulou, T. Xifilidis, K. E. Psannis, K. Siakavara, and Y. Ishibashi, "A survey on spectrum sensing algorithms for cognitive radio networks," in 2018 7th International Conference on Modern Circuits and Systems Technologies (MOCAST), 2018, pp. 1-4.

[26] B. Scheers, D. Teguig, and V. Le Nir, "Wideband spectrum sensing technique based on goodness-of-fit testing," in 2015 International Conference on Military Communications and Information Systems (ICMCIS), 2015, pp. 1-6.

[27] D. Teguig, B. Scheers, V. Le Nir, and F. Horlin, "Spectrum sensing method based on the likelihood ratio goodness of fit test under noise uncertainty," International Journal of Engineering Research and Technology (IJERT), vol. 3, pp. 488-494, 092014.

[28] T. J. O'Shea, T. Roy, and T. C. Clancy, "Over-the-air deep learning based radio signal classification," IEEE Journal of Selected Topics in Signal Processing, vol. 12, no. 1, pp. 168-179, Feb 2018.

[29] S. Rajendran, W. Meert, D. Giustiniano, V. Lenders, and S. Pollin, "Deep learning models for wireless signal classification with distributed lowcost spectrum sensors," IEEE Transactions on Cognitive Communications and Networking, pp. 1-1, 2018.

[30] S. Riyaz, K. Sankhe, S. Ioannidis, and K. Chowdhury, "Deep learning convolutional neural networks for radio identification," IEEE Communications Magazine, vol. 56, no. 9, pp. 146-152, Sep. 2018.

[31] K. He, X. Zhang, S. Ren, and J. Sun, "Deep residual learning for image recognition," in 2016 IEEE Conference on Computer Vision and Pattern Recognition (CVPR), 2016, pp. 770-778.

[32] J. Redmon, S. Divvala, R. Girshick, and A. Farhadi, "You only look once: Unified, real-time object detection," in 2016 IEEE Conference on Computer Vision and Pattern Recognition (CVPR), 2016, pp. 779-788.

[33] T. O'Shea, T. Roy, and T. C. Clancy, "Learning robust general radio signal detection using computer vision methods," in 2017 51st Asilomar Conference on Signals, Systems, and Computers, 2017, pp. 829-832.

[34] E. Fonseca, J. F. Santos, F. Paisana, and L. A. DaSilva, "Radio access technology characterisation through object detection," Computer Communications, vol. 168, pp. 12-19, 2021. [Online]. Available: https://www.sciencedirect.com/science/article/pii/S0140366420320272

[35] S. Couturier and D. Rauschen, "Energy detection based on long-term estimation of gaussian noise distribution," in 8th Karlsruhe Workshop on Software Radios, 2014.

[36] S. R. Kaiming He, Xiangyu Zhang and J. Sun, "Delving deep into rectifiers: Surpassing human-level performance on imagenet classification," ICCV, 2015.

[37] R. Huang, J. Pedoeem, and C. Chen, "Yolo-lite: A real-time object detection algorithm optimized for non-gpu computers," in 2018 IEEE International Conference on Big Data (Big Data), 2018, pp. 2503-2510.

[38] D. P. Kingma and J. Ba, "Adam: A method for stochastic optimization," CoRR, vol. abs/1412.6980, 2015.

[39] M. Abadi, A. Agarwal et al., "TensorFlow: Large-scale machine learning on heterogeneous systems," 2015, software available from tensorflow.org. [Online]. Available: https://www.tensorflow.org/

[40] "Horizon2020. The SafeShore project," In http://safeshore.eu, funded by the European Commission under the "Horizon 2020" program, grant agreement No 700643 . 


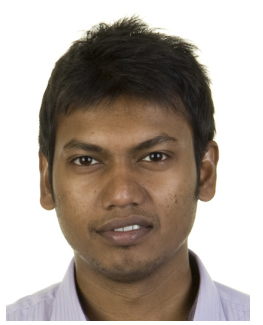

Sanjoy Basak received the M.Sc. degree in electrical engineering and information technology from Karlsruhe Institute of Technology, Karlsruhe, Germany, in 2016. He joined the Royal Military Academy, Belgium as a Researcher in 2016, where he is currently pursuing a joint doctoral study in association with KU Leuven in the Department of Electrical Engineering since 2019. His research interests include deep learning algorithms for wireless signal detection and classification.

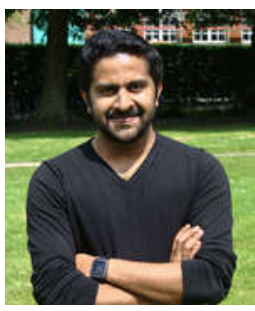

Sreeraj Rajendran received the master's degree in communication and signal processing from the Indian Institute of Technology Bombay, India, in 2013, and the Ph.D. degree from KU Leuven, Belgium, in 2019, where he is a Post-Doctoral Researcher with the Networked Systems Group. In 2013, he was a Senior Design Engineer with the Baseband Team, Cadence (Tensilica). He was also an ASIC Verification Engineer with Wipro Technologies from 2007 to 2010. His main research interests include machine learning algorithms for wireless spectrum awareness and low power wireless sensor networks.

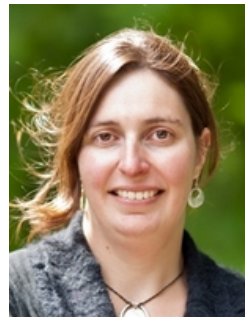

Sofie Pollin (Senior Member, IEEE) received the $\mathrm{Ph} . \mathrm{D}$. degree (Hons.) from KU Leuven, Leuven, Belgium, in 2006. From 2006 to 2008, she continued her research on wireless communication, energyefficient networks, cross-layer design, coexistence, and cognitive radio at UC Berkeley. In November 2008, she returned to IMEC, Leuven, to become a Principal Scientist in the Green Radio Team. Since 2012, she has been a tenure track Assistant Professor with the Department of Electrical Engineering, KU Leuven. Her research centers around networked systems that require networks that are ever more dense, heterogeneous, batterypowered, and spectrum constrained. Prof. Pollin is a fellow of the BAEF and Marie Curie.

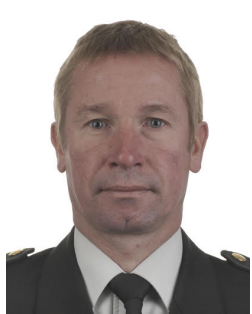

Bart Scheers was born in Rumst, Belgium, in November 1966. He received the M.S. degree in engineering, with a specialisation in communication from the Royal Military Academy, Brussels, Belgium in 1991 and a joint Ph.D. degree in 2001 from the Université catholique de Louvain, Ottignies-Louvain-la-Neuve, Belgium/Royal Military Academy, where he presented his Ph.D. dissertation on the use of ground penetrating radars in the field of humanitarian demining. After his studies, he was an Officer in a territorial signal unit of the Belgian Army. In 1994, he was an Assistant in the field of signal processing with the Royal Military Academy, where since 2003, he has been a Military Professor with the Communications Information Systems and Sensors (CISS) department, and is also the Director of the research unit on radio networks. His current domains of interest are mobile ad hoc networks (layers 2 and 3), cognitive radio, and internet of things. 